\title{
The physical meaning of transmission-line parameters in a full-wave theory
}

\author{
Ronald Rambousky $^{1}$, Jürgen Nitsch ${ }^{2}$, and Sergey Tkachenko ${ }^{2}$ \\ ${ }^{1}$ Bundeswehr Research Institute for Protective Technologies and \\ NBC Protection (WIS), Munster, Germany \\ ${ }^{2}$ Otto-von-Guericke University Magdeburg, Magdeburg, Germany \\ Correspondence to: Sergey Tkachenko (sergey.tkachenko@ovgu.de)
}

Received: 29 November 2015 - Revised: 10 May 2016 - Accepted: 21 May 2016 - Published: 28 September 2016

\begin{abstract}
In the potential-current representation, transmission-line parameters in the Transmission-Line Super Theory (TLST) do not have a direct physical meaning - they are gauge dependent, i.e.: they are different in the Lorenz and Coulomb gauge. However, they retain traces of their classical origin: They are constituted of capacitances and inductances for forward and backward running waves along the lines. Therefore their corresponding matrices are not symmetrical as in the case of classical transmission-line theory. In the charge-current representation the parameter matrices have a physical meaning: their elements consist of damping functions due to the non-uniformities of the lines and of the propagation functions along the lines, incorporating conductor and radiation losses. The transmission line parameters also contribute to the total radiated power of the lines. The attempt to quantize radiation locally, fails because radiation describes a long-range (integral) interaction, and therefore affects all conductor parts of all lines. However, it can be stated that at stronger inhomogeneities the local contributions to radiation increase, and are particularly recognizable along the risers.
\end{abstract}

\section{Introduction}

Haase and Nitsch (Haase and Nitsch, 2001) and Nitsch and Tkachenko (Nitsch and Tkachenko, 2010) have introduced a Maxwellian (Full-Wave) Theory, the Transmission-Line Super Theory (TLST), for a system of non-uniform transmission lines. The equations of these theories resemble those of classical transmission-line theory, but with a completely occupied parameter matrix. The new transmission line param- eters are complex-valued; they depend on frequency and on local coordinates. They can be represented in different representations, such as in the potential-current representation or in the charge-current representation, to name just two. Moreover, the parameters in the potential-current representation are gauge dependent, and they differ in the Coulomb gauge from those in the Lorenz gauge.

On the other hand, it was shown (Nitsch and Tkachenko, 2009) that the generalized transmission-line parameters contain all modes which propagate along the lines: TEM-modes, leaky modes, and radiation modes. Additionally, they are related to the (measurable) propagation and damping matrices in a second order differential equation for forward and backward running current waves. Based on the above facts, the question arises regarding the physical meaning of each parameter. In classical transmission-line theory they have the meaning of a capacitance and inductance per-unit length. This paper considers different aspects to the posed question: Sect. 2 presents a brief collection of the equations in the charge-current representation and in the potentialcurrent representation, as well as the parameters in their corresponding representation. In Sect. 3 the transmission-line parameters are formulated in both the Lorenz gauge and the Coulomb gauge. Section 4 shows the relation of the line parameters to the total radiated power, whereas Sect. 5 gives numerical examples for the individual contributions of the parameters in different sections of a line to the radiated power and for the propagation and damping functions of the considered line. Finally, Sect. 6 concludes this paper. 


\section{Transmission-line super theory equation in different representations}

For the notation in this paper the reader is referred to the publications (Nitsch et al., 2009), (Rambousky et al., 2014), and (Nitsch and Tkachenko, 2010). In Nitsch et al. (2009) it was shown that the Mixed Potential Integral Equations (MPIE) for thin and lossless multi-conductor lines could be cast into the form of generalized Telegrapher's equations, reading:

$$
\frac{\partial}{\partial l}\left[\begin{array}{c}
\boldsymbol{q}^{\prime}(l) \\
\boldsymbol{i}(l)
\end{array}\right]+j \omega\left[\begin{array}{cc}
\mathbf{P}_{11}(l) & \mathbf{P}_{12}(l) \\
\mathbf{1} & \mathbf{0}
\end{array}\right]\left[\begin{array}{c}
\boldsymbol{q}^{\prime}(l) \\
\boldsymbol{i}(l)
\end{array}\right]=\left[\begin{array}{c}
\boldsymbol{q}_{\mathrm{exc}}^{\prime \prime}(l) \\
\mathbf{0}
\end{array}\right]
$$

In this equation the parameter $l$ denotes a generalized length for all lines, $\boldsymbol{q}^{\prime}(l)$ is the charge vector per unit-length, the block matrices $\mathbf{P}_{11}(l)$ and $\mathbf{P}_{12}(l)$ are the length and frequency dependent complex-valued line-parameter matrices with units $\left[\mathbf{P}_{11}\right]=\mathrm{s} \mathrm{m}^{-1},\left[\mathbf{P}_{12}\right]=\mathrm{s}^{2} \mathrm{~m}^{-2}$, and $\boldsymbol{q}_{\mathrm{exc}}^{\prime \prime}(l)$ denotes an exterior distributed renormalized excitation source vector (see below). With the aid of the continuity Eq. (1) can be rewritten into a second order differential equation for the current vector $\boldsymbol{i}$ :

$$
\left(\frac{\partial^{2}}{\partial l^{2}}-\mathbf{D} \cdot \frac{\partial}{\partial l}-\Gamma^{2}\right) \cdot \boldsymbol{i}(l)=i_{\mathrm{S}}^{\prime \prime}
$$

Here the propagation function matrix $\boldsymbol{\Gamma}$ is, in general, a complex valued matrix, incorporating losses caused by conductor losses as well as by radiation. The matrix $\mathbf{D}$ describes damping along the line due to the reflections caused by nonuniformities (Nitsch et al., 2009). The matrices in Eq. (2) are related to the matrices in Eq. (1) as follows:

$$
\begin{aligned}
& \mathbf{D}=-j \omega \mathbf{P}_{11},[\mathbf{D}]=1 / \mathrm{m}, \\
& \quad \text { and } \boldsymbol{\Gamma}^{2}=-\omega^{2} \mathbf{P}_{12},[\boldsymbol{\Gamma}]=1 / \mathrm{m}
\end{aligned}
$$

Thus it can be stated that the parameter matrices in Eqs. (1) and (2) have a physical meaning and can be measured. In particular, they do not depend on gauge.

The solution of Eq. (1) is also known and results in:

$$
\begin{aligned}
{\left[\begin{array}{c}
\boldsymbol{q}^{\prime}(l) \\
\boldsymbol{i}(l)
\end{array}\right]=} & \mathcal{M}_{l_{0}}^{l}\{-j \omega \overline{\mathbf{P}}\}\left[\begin{array}{c}
\boldsymbol{q}^{\prime}\left(l_{0}\right) \\
\boldsymbol{i}\left(l_{0}\right)
\end{array}\right] \\
& +\int_{l_{0}}^{l} \mathcal{M}_{l^{\prime}}^{l}\{-j \omega \overline{\mathbf{P}}\} \cdot\left[\begin{array}{c}
\boldsymbol{q}_{\text {exc }}^{\prime \prime}\left(l^{\prime}\right) \\
\mathbf{0}
\end{array}\right] \mathrm{d} l^{\prime}
\end{aligned}
$$

The quantity $\mathcal{M}_{l_{0}}^{l}$ is the so-called matrizant or product integral (see Gantmacher, 1984). In order to obtain a solution of Eq. (4) one first has to know the parameter block matrix $\overline{\mathbf{P}}$ and subsequently calculate the (unknown) renormalized distributed sources $\boldsymbol{q}_{\text {exc }}^{\prime \prime}(l)$ from a given exterior field excitation $\mathbf{v}_{\text {exc }}^{\prime}$ of the lines. In the case of low frequencies $(k \rightarrow 0)$ this relation can be easily derived (see Rambousky et al., 2014). This example starts with one set of the MPIE (the other set is given by the continuity equation).

$$
\begin{aligned}
& \frac{1}{4 \pi \epsilon_{0}} \frac{\partial}{\partial l} \int_{0}^{L} \mathbf{G}_{\mathrm{C}}\left(l, l^{\prime}, k\right) \boldsymbol{q}^{\prime}\left(l^{\prime}\right) \mathrm{d} l^{\prime} \\
& \quad+j \omega \frac{\mu_{0}}{4 \pi} \int_{0}^{L} \mathbf{G}_{\mathrm{L}}\left(l, l^{\prime}, k\right) \boldsymbol{i}\left(l^{\prime}\right) \mathrm{d} l^{\prime}=\mathbf{v}_{\mathrm{exc}}^{\prime}(l)
\end{aligned}
$$

or in an even more compact form:

$$
\left[\begin{array}{ll}
j \omega \mathbf{1} & \mathbf{1} \frac{\partial}{\partial l}
\end{array}\right]_{(N, 2 N)} \int_{0}^{L} \overline{\mathbf{G}}\left(l, l^{\prime}, k\right)\left[\begin{array}{c}
\boldsymbol{q}^{\prime}\left(l^{\prime}\right) \\
\boldsymbol{i}\left(l^{\prime}\right)
\end{array}\right] \mathrm{d} l^{\prime}=\mathbf{v}_{\mathrm{exc}}^{\prime}(l),
$$

with the definition of the block matrix

$$
\overline{\mathbf{G}}\left(l, l^{\prime}, k\right):=\left[\begin{array}{cc}
\mathbf{0} & \frac{\mu_{0}}{4 \pi} \mathbf{G}_{\mathrm{L}}\left(l, l^{\prime}, k\right) \\
\frac{1}{4 \pi \epsilon_{0}} \mathbf{G}_{\mathrm{C}}\left(l, l^{\prime}, k\right) & \mathbf{0}
\end{array}\right]
$$

Now, in the low-frequency limit $(k \rightarrow 0)$ Eq. (5) results into a second order differential equation of the form:

$$
\begin{aligned}
& \left(\frac{\partial^{2}}{\partial l^{2}}+\mathbf{C}^{\prime}(l) \cdot \frac{\partial \mathbf{C}^{\prime-1}(l)}{\partial l} \frac{\partial}{\partial l}+\omega^{2} \mathbf{C}^{\prime}(l) \cdot \mathbf{L}^{\prime}(l)\right) \cdot \boldsymbol{i}(l) \\
& \quad=-j \omega \mathbf{C}^{\prime}(l) \mathbf{v}_{\mathrm{exc}}^{\prime}(l)
\end{aligned}
$$

Here the capacitance matrix per unit length $\mathbf{C}^{\prime}(l)$ and the inductance matrix per unit length $\mathbf{L}^{\prime}(l)$ are correlated to their corresponding Green's functions via:

$\mathbf{C}^{\prime}(l)=4 \pi \epsilon_{0}\left(\int_{0}^{L} \mathbf{G}_{\mathrm{C}}\left(l, l^{\prime}, 0\right) \mathrm{d} l^{\prime}\right)^{-1}$

$\mathbf{L}^{\prime}(l)=\frac{\mu_{0}}{4 \pi} \int_{0}^{L} \mathbf{G}_{\mathrm{L}}\left(l, l^{\prime}, 0\right) \mathrm{d} l^{\prime}$

Comparing Eq. (8) with Eqs. (5) and (2) one recognizes the following relations between the distributed sources:

$-j \omega \boldsymbol{q}_{\mathrm{exc}}^{\prime \prime}(l)=\boldsymbol{i}_{S}^{\prime \prime}(l) \approx-j \omega \mathbf{C}^{\prime}(l) \mathbf{v}_{\mathrm{exc}}^{\prime}(l)$

Here the first equal sign reflects the continuity equation, whereas the second (approximately equal) sign shows the connection between the source in the MPIE Eq. (5) and the source in Eq. (1) for the charge-current representation in the low frequency limit. This is an important intermediate result. Different from classical transmission-line theory one observes here that the line parameters are local dependent (but still real and gauge-independent), i.e., the lines are allowed to be non-uniform. 
For the general solution - without any restriction to frequencies - Eq. (4) is inserted for the block column vector $\left[\boldsymbol{q}^{\prime}\left(l^{\prime}\right) \boldsymbol{i}\left(l^{\prime}\right)\right]^{T}$ in Eq. (6), and after some rearrangements one arrives at:

$$
\begin{aligned}
\mathbf{I}_{21} \frac{\partial \boldsymbol{q}^{\prime}}{\partial l} & +\mathbf{I}_{22} \frac{\partial \boldsymbol{i}}{\partial l}+\left(j \omega \mathbf{I}_{11}+\frac{\partial \mathbf{I}_{21}}{\partial l}\right) \boldsymbol{q}^{\prime} \\
& +\left(j \omega \mathbf{I}_{12}+\frac{\partial \mathbf{I}_{22}}{\partial l}\right) \boldsymbol{i}+j \omega \mathbf{I}_{01}+\frac{\partial \mathbf{I}_{02}}{\partial l}=\mathbf{v}_{\mathrm{exc}}^{\prime}
\end{aligned}
$$

The matrices $\mathbf{I}_{i j}(i, j=1,2)$ of the block matrix $\overline{\mathbf{I}}$ and the block vector $\left[\mathbf{I}_{10}(l) \mathbf{I}_{20}(l)\right]^{T}$ are defined through:

$$
\begin{aligned}
& \overline{\mathbf{I}}(l):=\int_{0}^{L} \overline{\mathbf{G}}\left(l, l^{\prime}, k\right) \mathcal{M}_{l}^{l^{\prime}}(-j \omega \overline{\mathbf{P}}) \mathrm{d} l^{\prime} \\
& {\left[\mathbf{I}_{10}(l) \mathbf{I}_{20}(l)\right]^{T}:=\int_{0}^{L} \overline{\mathbf{G}}\left(l, l^{\prime}, k\right)} \\
& \quad \int_{l}^{l^{\prime}} \mathcal{M}_{l^{\prime \prime}}^{l^{\prime}}\{-j \omega \overline{\mathbf{P}}\} \cdot\left[\boldsymbol{q}_{\mathrm{exc}}^{\prime \prime}\left(l^{\prime}\right) \mathbf{0}\right] \mathrm{d} l^{\prime \prime} \mathrm{d} l^{\prime}
\end{aligned}
$$

Equation (11) together with the continuity equation can now be written in the desired form Eq. (1) where one obtains for the parameter block matrix $\overline{\mathbf{P}}$ and the unknown $\boldsymbol{q}_{\mathrm{exc}}^{\prime \prime}$ :

$\overline{\mathbf{P}}=$

$\left[\begin{array}{cc}\mathbf{I}_{21}^{-1}\left(\mathbf{I}_{11}-\mathbf{I}_{22}\right)+\frac{1}{j \omega} \frac{\partial \mathbf{I}_{21}}{\partial l} & \mathbf{I}_{21}^{-1}\left(\mathbf{I}_{12}+\frac{1}{j \omega} \frac{\partial \mathbf{I}_{22}}{\partial l}\right) \\ \mathbf{1} & \mathbf{0}\end{array}\right]$

$\boldsymbol{q}_{\mathrm{exc}}^{\prime \prime}=\mathbf{I}_{21}^{-1}\left(\mathbf{v}_{\mathrm{exc}}^{\prime}-j \omega \mathbf{I}_{10}-\frac{\partial \mathbf{I}_{20}}{\partial l}\right)$

At this point it seems appropriate to give a brief interpretation of the previous results. The starting point of the above considerations was the MPIE Eq. (5). From this it could be demonstrated that - for low frequencies - it obeyed a wave Eq. (8) for the current. Assuming now that for any frequencies the current fulfills a wave Eq. (2) (forward - and backward running waves for the homogeneous solution), then one obtains (again with the aid of the continuity equation) the generalized line equations in the charge-current representation Eq. (1). However, the line parameters and the sources are still unknown in this equation. These unknowns are eventually derived by insertion of the solution ansatz Eq. (4) in Eq. (1) and are represented by Eq. (13a, b).

The iterative solution procedures of Eq. $(13 \mathrm{a}, \mathrm{b})$ are de-

\begin{tabular}{|c|}
\hline$\overline{\mathbf{I}}^{(0)}(l) \rightarrow \overline{\mathbf{P}}^{(0)}(l) \rightarrow \mathcal{M}_{l_{0}}^{l}\left\{-\jmath \omega \overline{\mathbf{P}}^{(0)}\right\} \rightarrow \overline{\mathbf{I}}^{(1)}(l) \rightarrow \overline{\mathbf{P}}^{(1)}(l)$ \\
\hline Zeroth iteration step: \\
\hline 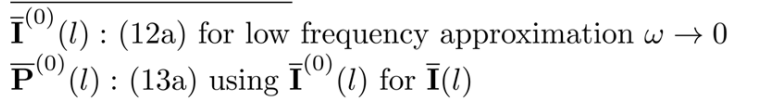 \\
\hline First iteration step: \\
\hline$\overline{\overline{\mathbf{I}}^{(1)}(l) \text { : (12a) using }} \overline{\mathbf{P}}^{(0)}(l)$ for $\overline{\mathbf{P}}(l)$ \\
\hline $\mathbf{P}(l):(13 a)$ using $\mathbf{T}(l)$ for $\mathbf{I}(l)$ \\
\hline
\end{tabular}
scribed in detail in Nitsch et al. (2009) an can be visualized in the flow-diagram shown in Fig. 1.

The parameters are derived from the homogeneous problem of Eq. (1), i.e., without exterior distributed sources.
Figure 1. Flow-diagram of the iterative solution procedure for the TLST parameter matrix.

Therefore, they do not depend on those sources or on boundary conditions. After knowing the parameters in an appropriate approximation, the renormalized sources are calculated. The enforced (by the exterior distributed sources) solution of Eq. (2) (besides the two fundamental solutions) is hidden in the renormalized sources. The renormalization of the exterior source $\mathbf{v}_{\mathrm{exc}}^{\prime}$ in the MPIE is the price which has to be paid for the fact that the parameter matrix $\overline{\mathbf{P}}$ is solely determined in the absence of distributed sources (Nitsch and Tkachenko, 2010).

In many cases it is convenient to deal with voltages or potentials instead of a per-unit length charge. For instance, if the transmission line is embedded into a circuit or if other elements are connected to the line, the voltages at the terminals must be known to perform the calculations. The connection between the potential vector $\varphi(l)$ and the special Greens function $\mathbf{G}_{\mathrm{C}}\left(l, l^{\prime}\right)$ is given by:

$\boldsymbol{\varphi}(l)=\frac{1}{4 \pi \epsilon_{0}} \int_{0}^{L} \mathbf{G}_{\mathrm{C}}\left(l, l^{\prime}\right) \boldsymbol{q}^{\prime}\left(l^{\prime}\right) \mathrm{d} l^{\prime}$

Using the above equations this expression becomes

$\left[\begin{array}{c}\boldsymbol{\varphi} \\ \boldsymbol{i}\end{array}\right]=\left[\begin{array}{cc}\mathbf{I}_{21} & \mathbf{I}_{22} \\ \mathbf{0} & \mathbf{1}\end{array}\right]\left[\begin{array}{c}\boldsymbol{q}^{\prime} \\ \boldsymbol{i}\end{array}\right]+\left[\begin{array}{c}\mathbf{I}_{20} \\ \mathbf{0}\end{array}\right]$

Equation (15) is valid along the entire line. Therefore, the telegrapher equations (Eq. 1) can be easily converted into the potential-current representation. This is the subject of the next section.

\section{The transmission-line parameters in the Lorenz and Coulomb gauge}

Taking the partial derivative of Eq. (15) with respect to $l$, using Eq. (1) for the derivative of the column vector $\left[\boldsymbol{q}^{\prime} \boldsymbol{i}\right]^{T}$ and replacing $\boldsymbol{q}^{\prime}$ using Eq. (15) then Eq. (1) is converted into the desired potential-current representation in the Lorenz gauge:

$$
\frac{\partial}{\partial l}\left[\begin{array}{c}
\boldsymbol{\varphi}(l) \\
\boldsymbol{i}(l)
\end{array}\right]+j \omega \overline{\mathbf{P}}^{*}(l)\left[\begin{array}{c}
\boldsymbol{\varphi}(l) \\
\boldsymbol{i}(l)
\end{array}\right]=\left[\begin{array}{c}
\boldsymbol{\varphi}_{S}^{\prime}(l) \\
\boldsymbol{i}_{S}^{\prime}(l)
\end{array}\right],
$$


where

$\overline{\mathbf{P}}^{*}=\left[\begin{array}{cc}\mathbf{I}_{11} \mathbf{I}_{21}^{-1} & \mathbf{I}_{12}-\mathbf{I}_{11} \mathbf{I}_{21}^{-1} \mathbf{I}_{22} \\ \mathbf{I}_{21}^{-1} & -\mathbf{I}_{21}^{-1} \mathbf{I}_{22}\end{array}\right]$

and

$\left[\begin{array}{c}\boldsymbol{\varphi}_{S}^{\prime}(l) \\ \boldsymbol{i}_{S}^{\prime}(l)\end{array}\right]=\left[\begin{array}{c}\mathbf{v}_{\mathrm{exc}}^{\prime}+j \omega\left(\mathbf{I}_{11} \mathbf{I}_{21}^{-1} \mathbf{I}_{20}-\mathbf{I}_{10}\right) \\ j \omega \mathbf{I}_{21}^{-1} \mathbf{I}_{20}\end{array}\right]$

Equations (16) and (17) provide the proof that the MPI equations can be cast into the form of telegrapher equations. However, one has to calculate the parameters using Eq. (17a) and renormalized sources have to be used also for the current. These renormalized sources are completely different from both classical TLT and from the low frequency approach, where the current source appears as zero. This important aspect was not discussed in the paper of Shen et al. (2011). In that paper the authors used incorrect generalized transmission-line equations and, consequently (in general) obtained false results.

The next step refers to the derivation of the line parameters in the Coulomb gauge. In order to indicate the potentials in their special gauge, they are indexed accordingly. The MPIE in the Lorenz gauge read:

$\frac{\partial \boldsymbol{\varphi}_{\text {Lor }}}{\partial l}+j \omega \frac{\mu_{0}}{4 \pi} \int_{0}^{L} \mathbf{G}_{\mathrm{L}}\left(l, l^{\prime}, k\right) \boldsymbol{i}\left(l^{\prime}\right) \mathrm{d} l^{\prime}=\mathbf{0}$

and

$\boldsymbol{\varphi}_{\text {Lor }}(l)=-\frac{1}{j \omega 4 \pi \epsilon_{0}} \int_{0}^{L} \mathbf{G}_{\mathrm{C}}\left(l, l^{\prime}, k\right) \frac{\partial \boldsymbol{i}\left(l^{\prime}\right)}{\partial l} \mathrm{~d} l^{\prime}$

Introducing the potential in the Coulomb gauge

$\boldsymbol{\varphi}_{\text {Coul }}(l)=-\frac{1}{j \omega 4 \pi \epsilon_{0}} \int_{0}^{L} \mathbf{G}_{\mathrm{C}}\left(l, l^{\prime}, 0\right) \frac{\partial \boldsymbol{i}\left(l^{\prime}\right)}{\partial l} \mathrm{~d} l^{\prime}$

one rewrites the Lorenz potential as

$$
\begin{aligned}
\boldsymbol{\varphi}_{\text {Lor }}(l)= & \boldsymbol{\varphi}_{\text {Coul }}(l)-\frac{1}{j \omega 4 \pi \epsilon_{0}} \\
& \cdot \int_{0}^{L}\left\{\mathbf{G}_{\mathrm{C}}\left(l, l^{\prime}, k\right)-\mathbf{G}_{\mathrm{C}}\left(l, l^{\prime}, 0\right)\right\} \frac{\partial \boldsymbol{i}\left(l^{\prime}\right)}{\partial l} \mathrm{~d} l^{\prime}
\end{aligned}
$$

The derivative of Eq. (20) with respect to $l$ (together with Eq. 18a) gives:

$$
\begin{array}{r}
\frac{\partial \boldsymbol{\varphi}_{\mathrm{Coul}}}{\partial l}-\frac{1}{j \omega 4 \pi \epsilon_{0}} \int_{0}^{L} \frac{\partial}{\partial l}\left\{\mathbf{G}_{\mathrm{C}}\left(l, l^{\prime}, k\right)-\mathbf{G}_{\mathrm{C}}\left(l, l^{\prime}, 0\right)\right\} \\
\cdot \frac{\partial \boldsymbol{i}\left(l^{\prime}\right)}{\partial l} \mathrm{~d} l^{\prime}+j \omega \frac{\mu_{0}}{4 \pi} \int_{0}^{L} \mathbf{G}_{\mathrm{L}}\left(l, l^{\prime}, k\right) \boldsymbol{i}\left(l^{\prime}\right) \mathrm{d} l^{\prime}=\mathbf{0}
\end{array}
$$

Equations (19) and (21) constitute the (homogeneous) MPIE in the Coulomb gauge. The next steps follow the concept (Nitsch and Tkachenko, 2005) applied to calculate the line parameters of first order in the Lorenz gauge using a perturbation theory. Here analogue steps are performed, however, with the restriction to one conductor to simplify the derivation. The generalization to $N$ conductors is discussed in Nitsch and Tkachenko (2010).

The fundamental solutions of the homogeneous MPIE in classical TLT for the current are forward- and backward running waves (after the first iteration):

$i_{1,2}^{(1)}(l)=e^{\mp j k l} \quad$ and $\quad \frac{\partial i_{1,2}^{(1)}(l)}{\partial l}=\mp j k e^{\mp j k l}$

These results are inserted into Eqs. (19) and (21) yielding:

$\varphi_{1,2 \text { Coul }}^{(1)}(l)=-\frac{1}{j \omega 4 \pi \epsilon_{0}} \int_{0}^{L} G_{\mathrm{C}}\left(l, l^{\prime}, 0\right) \frac{\partial i_{1,2}^{(1)}\left(l^{\prime}\right)}{\partial l} \mathrm{~d} l^{\prime}$

and

$$
\begin{gathered}
\frac{\partial \varphi_{1,2 \text { Coul }}^{(1)}(l)}{\partial l}=\frac{1}{j \omega 4 \pi \epsilon_{0}} \int_{0}^{L} \frac{\partial}{\partial l}\left\{G_{\mathrm{C}}\left(l, l^{\prime}, k\right)-G_{\mathrm{C}}\left(l, l^{\prime}, 0\right)\right\} \\
\cdot \frac{\partial i_{1,2}^{(1)}\left(l^{\prime}\right)}{\partial l} \mathrm{~d} l^{\prime}-j \omega \frac{\mu_{0}}{4 \pi} \int_{0}^{L} G_{\mathrm{L}}\left(l, l^{\prime}, k\right) i_{1,2}^{(1)}\left(l^{\prime}\right) \mathrm{d} l^{\prime}
\end{gathered}
$$

From these fundamental solutions in the Coulomb gauge the line parameters in this gauge can be calculated via the relation (Nitsch and Tkachenko, 2010)

$$
\left[\mathbf{X}^{(1)}\right]=\left[\begin{array}{cc}
\varphi_{1}^{(1)}(l) & \varphi_{2}^{(1)}(l) \\
i_{1}^{(1)}(l) & i_{2}^{(1)}(l)
\end{array}\right]
$$

and

$\overline{\mathbf{P}}_{\text {Coul }}^{(1)}=-\frac{1}{j \omega} \frac{\partial\left[\mathbf{X}^{(1)}\right]}{\partial l}\left[\mathbf{X}^{(1)}\right]^{-1}$

After some straightforward calculations one obtains the parameter matrix in the first order approximation:

$$
\begin{aligned}
P_{11 \text { Coul }}^{(1)}(l) & =c_{0}\left[{\widetilde{L^{\prime}}}_{+}^{(1)}(l)-{\widetilde{L^{\prime}}}_{-}^{(1)}(l)\right] \\
& \cdot\left\{\left[C_{+}^{\prime(0)(1)}(l)\right]^{-1}+\left[C_{-}^{\prime(0)(1)}(l)\right]^{-1}\right\}^{-1}
\end{aligned}
$$

$$
\begin{aligned}
& P_{12 \text { Coul }}^{(l)}(l) \\
& \quad\left\{{\widetilde{L^{\prime}}}_{+}^{(1)}(l)\left[C_{-}^{\prime(0)(1)}(l)\right]^{-1}+{\widetilde{L^{\prime}}}_{-}^{(1)}(l)\left[C_{+}^{\prime(0)(1)}(l)\right]^{-1}\right\} \\
& \cdot\left\{\left[C_{+}^{\prime(0)(1)}(l)\right]^{-1}+\left[C_{-}^{\prime(0)(1)}(l)\right]^{-1}\right\}^{-1} \\
& P_{21 \text { Coul }}^{(1)}(l)=2\left\{\left[C_{+}^{\prime(0)(1)}(l)\right]^{-1}+\left[C_{-}^{\prime(0)(1)}(l)\right]^{-1}\right\}
\end{aligned}
$$




$$
\begin{aligned}
P_{22 \mathrm{Coul}}^{(1)}(l) & =c_{0}^{-1}\left\{\left[{C^{\prime}}_{-}^{(0)(1)}(l)\right]^{-1}-\left[{C^{\prime}}_{+}^{(0)(1)}(l)\right]^{-1}\right\} \\
& \cdot\left\{\left[{C^{\prime}(0)(1)}_{+}(l)\right]^{-1}+\left[{C^{\prime}}_{-}^{(0)(1)}(l)\right]^{-1}\right\}^{-1}
\end{aligned}
$$

In Eqs. (26) through (29) the following expressions for the approximate inductance and capacitance were used:

$$
\begin{aligned}
\widetilde{L}_{ \pm}^{(1)}(l)= & \frac{\mu_{0}}{4 \pi} \int_{0}^{L}\left[\mp \frac{j}{k} \frac{\partial}{\partial l}\left(G_{\mathrm{C}}\left(l, l^{\prime}, k\right)-G_{\mathrm{C}}\left(l, l^{\prime}, 0\right)\right)\right. \\
& \left.+G_{\mathrm{L}}\left(l, l^{\prime}, k\right)\right] e^{\mp j k\left(l^{\prime}-l\right)} \mathrm{d} l^{\prime}
\end{aligned}
$$

$$
C_{ \pm}^{\prime(0)(1)}(l)=4 \pi \epsilon_{0}\left\{\int_{0}^{L} G_{\mathrm{C}}\left(l, l^{\prime}, 0\right) e^{\mp j k\left(l^{\prime}-l\right)} \mathrm{d} l^{\prime}\right\}^{-1}
$$

As can be seen, the parameters consist of the inductances and capacitances of forward and backward propagating waves along the lines. In general these quantities are different for the two waves, except in the case of lines with a high local symmetry, such as an infinite parallel line above ground. The diagonal parameter matrix elements are differences of the inductances and capacitances for forward and backward running waves, respectively. For both wave types, if these elements are equal they will vanish. Analogue arguments also hold for the parameter in the Lorenz gauge.

However, the parameters in the Coulomb gauge differ from those in the Lorenz gauge. The denominators of the Coulomb parameters are all real whereas the parameters which contain inductance terms are complex-valued. Due to the instantaneous relation of potential and charge in the Coulomb gauge, the parameter $P_{21 \mathrm{Coul}}^{(1)}(l)$ is pure real (Kazemzadeh and Mathis, 2009) and therefore does not contribute to radiation. This is different for parameter $P_{12 \mathrm{Coul}}^{(1)}(l)$ which is pure imaginary and therefore contributes to radiation. A comparison of parameters in both gauges for an infinite lossless TL above conducting ground was given by Nitsch and Tkachenko (2004). The next section deals with the role of the parameters in the Lorenz gauge in the radiated power of a transmission line.

\section{Radiated power of a non-uniform transmission-line and contribution of each individual conductor segment to the radiated power}

With the knowledge of the parameter matrix (in the Lorenz gauge) $\overline{\mathbf{P}}^{*(1)}(l, \omega)$ as a function of the arc length $l$ (or generalized length parameter for a multi-wire configuration) and frequency $\omega$ one can calculate the total radiated power of the line using the formula (Nitsch and Tkachenko, 2010)

$$
\begin{aligned}
P_{\operatorname{rad}}(\omega) & =j \frac{\omega}{4} \int_{0}^{L}\left[\boldsymbol{\varphi}^{+}\left(\mathbf{P}_{22}^{*(1)}-\mathbf{P}_{11}^{*(1)+}\right) \boldsymbol{i}\right. \\
& +\boldsymbol{i}^{+}\left(\mathbf{P}_{11}^{*(1)}-\mathbf{P}_{22}^{*(1)+}\right) \boldsymbol{\varphi}+\boldsymbol{i}^{+}\left(\mathbf{P}_{12}^{*(1)}-\mathbf{P}_{12}^{*(1)+}\right) \boldsymbol{i} \\
& \left.+\boldsymbol{\varphi}^{+}\left(\mathbf{P}_{21}^{*(1)}-\mathbf{P}_{21}^{*(1)+}\right) \boldsymbol{\varphi}\right] \mathrm{d} l
\end{aligned}
$$

Here the quantities $\boldsymbol{i}^{+}$and $\varphi^{+}$denote the transposed complex conjugate values of the current and potential vectors $i$ and $\boldsymbol{\varphi}$. The sub matrices $\mathbf{P}_{i j}^{*(1)+}$ are the transposed complex conjugate sub matrices $\mathbf{P}_{i j}^{*(1)}$. If one reduces the number of conductors to one line (plus reference) then the radiation formula becomes:

$$
\begin{aligned}
P_{\operatorname{rad}}(\omega)=-\frac{\omega}{2} \int_{0}^{L} & {\left[\Im\left(P_{12}^{*(1)}\right)|i|^{2}+\Im\left(P_{21}^{*(1)}\right)|\varphi|^{2}\right.} \\
+ & \left.\left(\varphi\left(P_{11}^{*(1)}-P_{22}^{*(1) \dagger}\right) i^{\dagger}\right)\right] \mathrm{d} l
\end{aligned}
$$

The sign $\dagger$ stands for the complex conjugate of a complex scalar. For a numerical calculation of Eqs. (32) and (33) the integral along the whole arc-length $L$ is replaced by a summation over all segments of the line. Assume the line is divided into $N_{\text {seg }}$ segments. Then $l_{j}$ denotes the arc length of the center position of the $j$ th line segment with length $\Delta_{j}$. For the radiated power one gets:

$$
\begin{aligned}
& P_{\mathrm{rad}}(\omega)=-\frac{\omega}{2} \sum_{j=1}^{N_{\mathrm{seg}}}\left[\Im\left(P_{12}^{*(1)}\left(l_{j}, \omega\right)\right)\left|i\left(l_{j}, \omega\right)\right|^{2}\right. \\
& +\Im\left(P_{21}^{*(1)}\left(l_{j}, \omega\right)\right)\left|\varphi\left(l_{j}, \omega\right)\right|^{2} \\
& \left.+\Im\left(\varphi\left(l_{j}, \omega\right)\left(P_{11}^{*(1)}\left(l_{j}, \omega\right)-P_{22}^{*(1) \dagger}\left(l_{j}, \omega\right)\right) i^{\dagger}\left(l_{j}, \omega\right)\right)\right] \Delta_{j}
\end{aligned}
$$

It is well known that radiation is an integral problem. If one, however, assumes for a moment that the radiation of individual line segments would have a meaning, then it would make sense to calculate the radiated power of these segments. Especially at strong inhomogenities of the conductor one would expect a stronger radiation than in largely homogeneous zones (experimentally shown in Schwark et al., 2008). The radiated power resulting from the $j$ th line segment and for frequency $\omega$ then would be:

$$
\begin{aligned}
& P_{\mathrm{rad}}(j, \omega)=-\frac{\omega}{2}\left[\Im\left(P_{12}^{*(1)}\left(l_{j}, \omega\right)\right)\left|i\left(l_{j}, \omega\right)\right|^{2}\right. \\
& +\Im\left(P_{21}^{*(1)}\left(l_{j}, \omega\right)\right)\left|\varphi\left(l_{j}, \omega\right)\right|^{2} \\
& \left.+\Im\left(\varphi\left(l_{j}, \omega\right)\left(P_{11}^{*(1)}\left(l_{j}, \omega\right)-P_{22}^{*(1) \dagger}\left(l_{j}, \omega\right)\right) i^{\dagger}\left(l_{j}, \omega\right)\right)\right] \Delta_{j}
\end{aligned}
$$

If one identifies the off-diagonal elements of the parameter matrix with inductance and capacitance per unit-length, respectively then the summands of Eq. (35) could be identified 


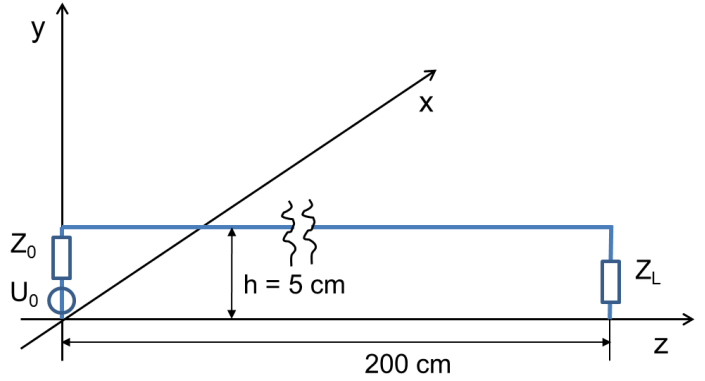

Figure 2. Parallel line above PEC ground with risers at each end.

as:

$P_{\text {rad,ind }}(j, \omega)=-\frac{\omega}{2} \Im\left(P_{12}^{*(1)}\left(l_{j}, \omega\right)\right)\left|i\left(l_{j}, \omega\right)\right|^{2} \Delta_{j}$

$P_{\text {rad,cap }}(j, \omega)=-\frac{\omega}{2} \mathfrak{\Im}\left(P_{21}^{*(1)}\left(l_{j}, \omega\right)\right)\left|\varphi\left(l_{j}, \omega\right)\right|^{2} \Delta_{j}$

In addition the radiated part of the diagonal parameters then becomes:

$$
\begin{aligned}
& P_{\text {rad,diag }}(j, \omega)=-\frac{\omega}{2} \Delta_{j} \\
& \cdot \Im\left(\varphi\left(l_{j}, \omega\right)\left(P_{11}^{*(1)}\left(l_{j}, \omega\right)-P_{22}^{*(1) \dagger}\left(l_{j}, \omega\right)\right) i^{\dagger}\left(l_{j}, \omega\right)\right)
\end{aligned}
$$

By analyzing the proportions Eqs. (36) through (38) to the radiated power of a segment one could determine to what extent the individual parameters contribute.

\section{Radiation, damping and propagation function for two simple conductor configurations - numerical examples}

The first line configuration starts with a parallel conductor above perfectly electrical conducting (PEC) ground, with two risers terminated with $50 \mathrm{Ohms}$ at both ends and fed by a $1 \mathrm{~V}$ source (see Fig. 2) on the left side. The dimensions of the conductor parts are specified.

Figure 3 displays the total radiated power in the frequency range between $100 \mathrm{MHz}$ and $1 \mathrm{GHz}$ calculated using Eq. (34). One recognizes clearly the resonances of the conductor and an increase of the radiated power with increasing frequencies.

Figures 4 and 5 show the radiation contributions of the individual conductor segments for a frequency of $1 \mathrm{GHz}$.

One can see that most radiation $P_{\mathrm{rad}}(j, \omega)$ takes place - as expected - at the ends of the conductor. The oscillating capacitive contribution $P_{\text {rad,cap }}(j, \omega)$ always stays in the negative regime, whereas the inductive part $P_{\text {rad,ind }}(j, \omega)$ oscillates in the positive regime, in phase with the capacitive part. In the asymptotic region of the conductor there is nearly no radiation. This is indicated by a pure oscillation of the power

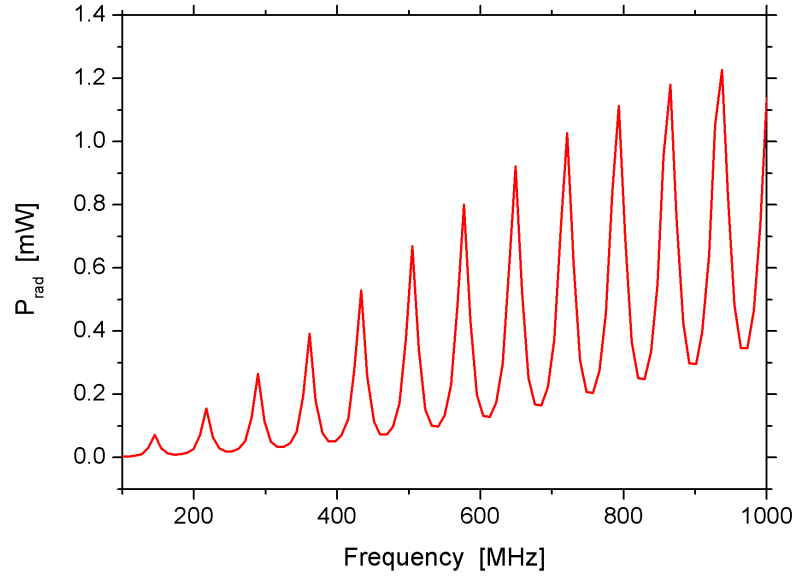

Figure 3. Total radiated power in the frequency range of $100 \mathrm{MHz}$ to $1 \mathrm{GHz}$.

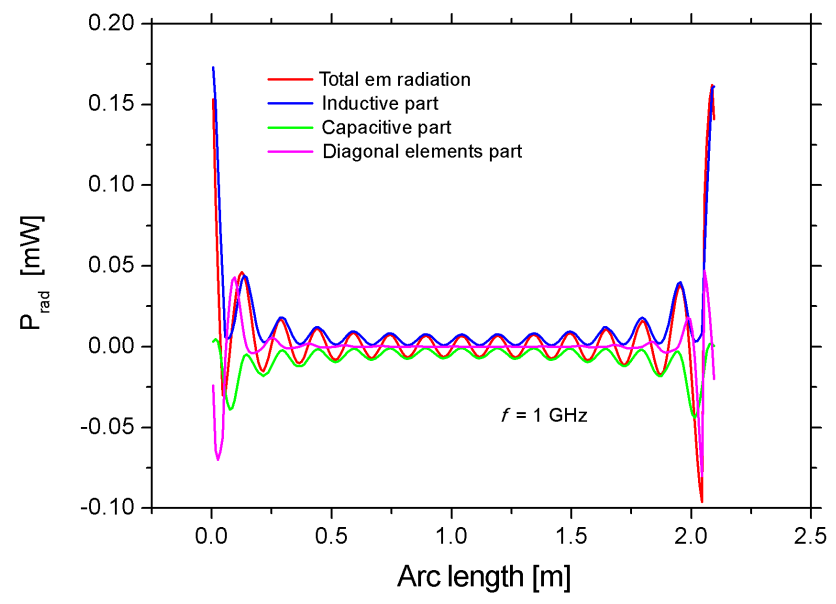

Figure 4. Different radiation contributions along the line from Fig. 2 at a frequency of $1 \mathrm{GHz}$.

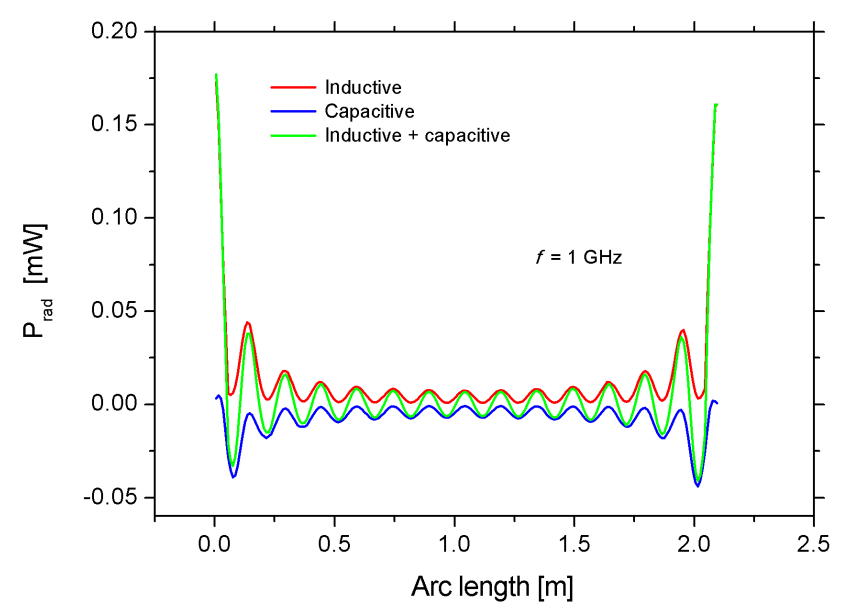

Figure 5. Capacitive and inductive radiation contributions along the line from Fig. 2 at a frequency of $1 \mathrm{GHz}$. 


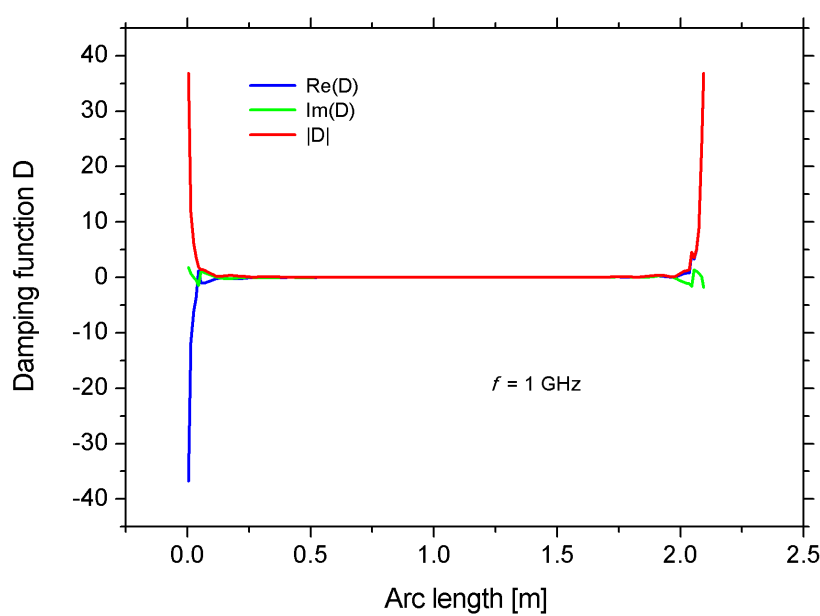

Figure 6. Damping function $D$ along the line from Fig. 2 at a frequency of $1 \mathrm{GHz}$.

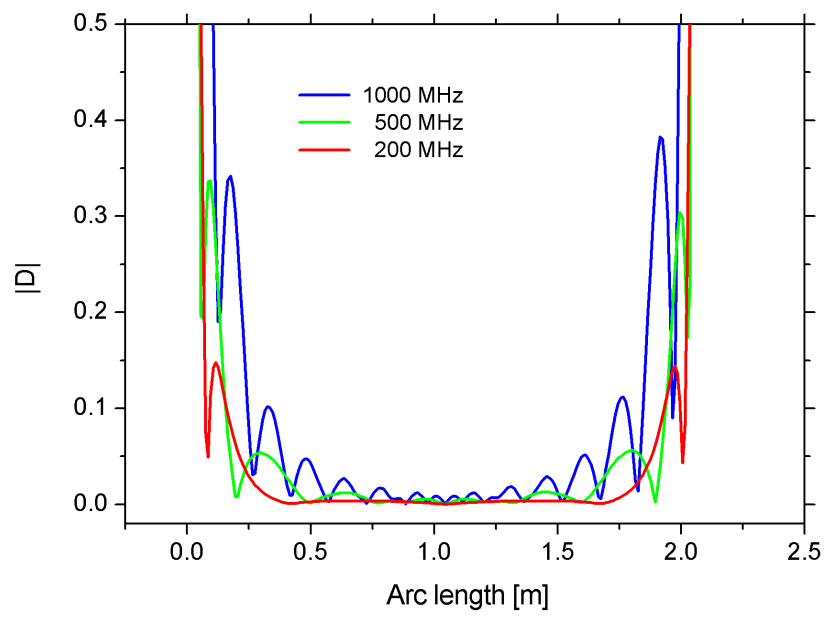

Figure 7. Magnitude of the damping function $|D|$ along the line from Fig. 2 for the frequencies $100,500 \mathrm{MHz}$ and $1 \mathrm{GHz}$.

around the local axis, showing that all energy is transported along this part of the line. The off-diagonal elements act essentially at the ends of the conductor. Note that radiation is an integral problem. This means that local, individual emission proportions do not have any physical meaning. However, they identify those portions of the line, where more or less radiation occurs. The observable quantities, damping function $D$ and propagation function $\Gamma$, are represented in the following figures.

Figures 6 and 7 represent the damping function $D$ for different scales of the $y$ axis.

Obviously, damping almost vanishes in the asymptotic regime of the line. It is strongest at the ends of the line, due to the strong change of the capacitance (see Eq. 8). And, as can be seen in Fig. 7, it increases with frequency.

It might be interesting to compare the shape of the damping function with that of the radiated power stemming from

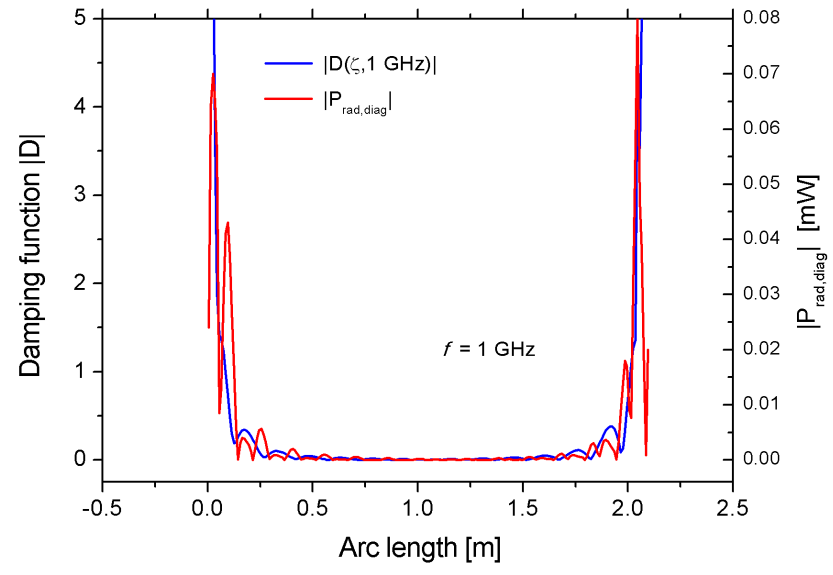

Figure 8. Comparison of the graphs for the damping function $|D|$ and for the radiated power of the diagonal elements $\left|P_{\text {rad,diag }}\right|$ (for the line from Fig. 2 at a frequency of $1 \mathrm{GHz}$ ).

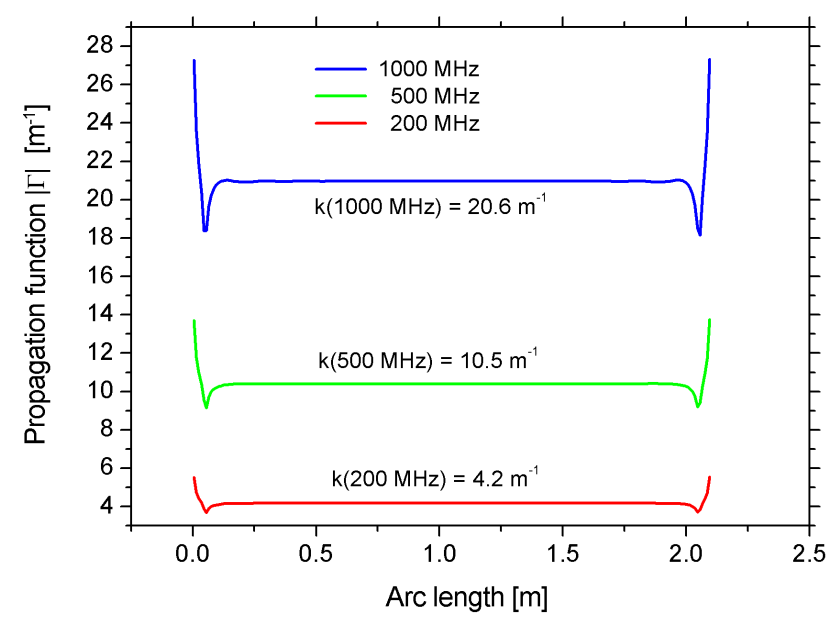

Figure 9. The magnitude of the propagation function $|\Gamma|$ for different frequencies (for the line from Fig. 2).

the diagonal parameter elements. In both cases the local change of the line configuration is important. In Fig. 8 one can see the similarity of both shapes.

The second measurable quantity is the propagation function $\Gamma$ which is represented in Fig. 9 for different frequencies. In the asymptotic region of the line the current waves propagate with the velocity of light. However, in the vicinity of the ends an interesting behavior is observed: The current waves (apparently) become faster than the speed of light $c$. This happens due to the radiation coupling between the riser and the horizontal part of the line. This coupling path is shorter than the path for the current waves through the end of the conductor and the riser. But, finally, the current waves are stopped along the risers and are reflected at the ends.

The next numerical example deals with a parallel line with two risers and a central local scatterer above PEC ground, 


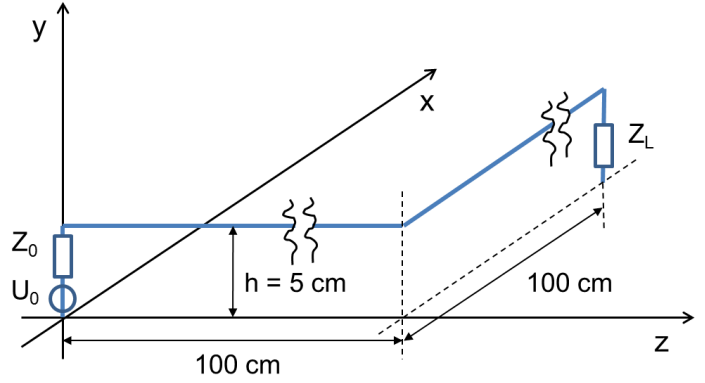

Figure 10. Parallel line over conducting ground with two risers and a central local scatterer $\left(90^{\circ}\right.$ bend $)$.

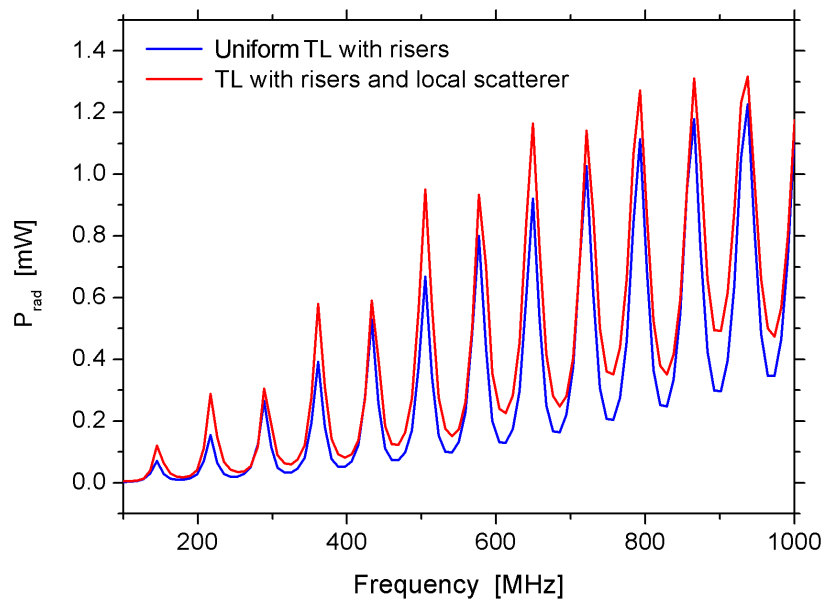

Figure 11. Total radiated power for a parallel line with and without a central local scatterer $\left(90^{\circ}\right.$ bend).

terminated at both ends with $50 \mathrm{Ohms}$ (see Fig. 10) and fed by a voltage source of $1 \mathrm{~V}$.

The bend in the line increases the radiated power (red curve in Fig. 11) compared to the emitted power of the line without bend (blue curve in Fig. 11).

Figure 12 shows the individual contributions of the line segments to the radiated power. The graphs look similar to those of Fig. 4, except around the central bend. There the radiated power increases slightly.

Similar arguments hold for the damping function $D$ of Fig. 13. An increased damping can be noticed at the ends as well as at the bend. This becomes more obvious in Fig. 14 where the damping function is represented again, but with a finer scale of the $y$ axis and for different frequencies. The damping at the central scatterer increases with increasing frequency as would be expected.

In Fig. 15 it is shown again that at those locations where damping becomes stronger the radiation contribution of the diagonal parameter elements also increases. Finally, Fig. 16 displays the graphs showing the magnitude of the propagation function $\Gamma$ for the frequencies $100,500 \mathrm{MHz}$ and $1 \mathrm{GHz}$.

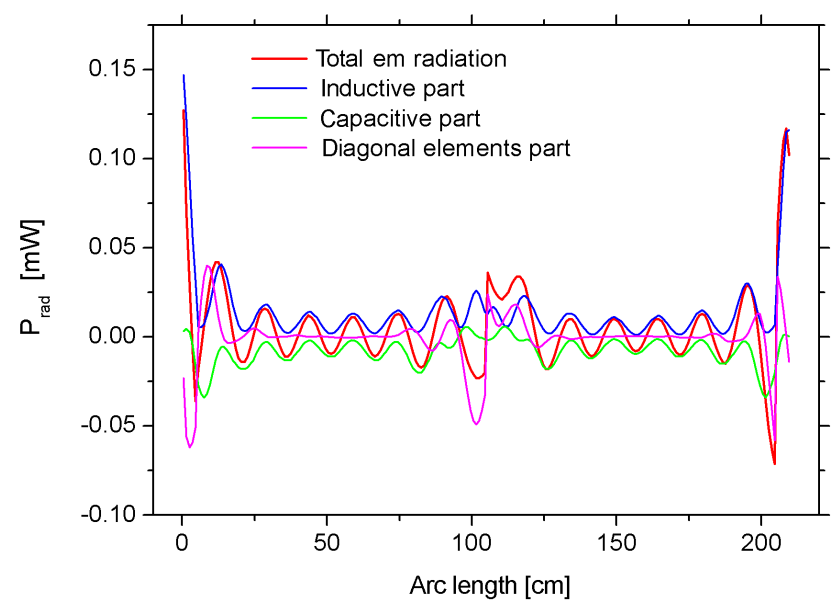

Figure 12. Different radiation contributions along the line from Fig. 10 for a frequency of $1 \mathrm{GHz}$ (total, inductive, capacitive, contribution of the diagonal elements).

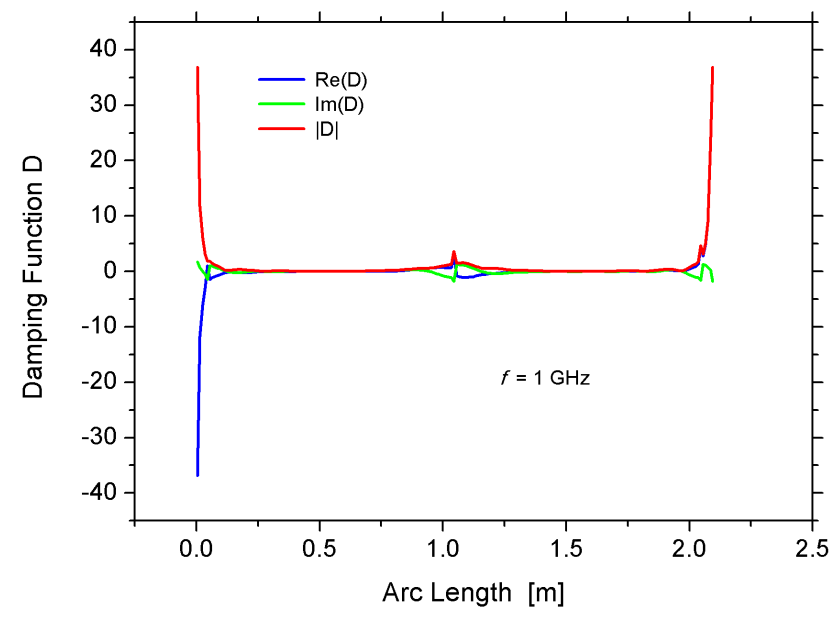

Figure 13. Damping function $D$ along the line with central local scatterer.

As in the previous line configuration, the current waves propagate with the speed of light $c$ in the asymptotic regions. Close to the risers and around the area of the bend the waves seem to run faster than $c$. Again, this happens because of the radiation interaction between line elements in the vicinity of the bends. The propagation path for the radiated power between the line elements near the sharp bend is shorter than the path for the current waves flowing through the bend. Therefore, the current induced by this radiation already occurs a little earlier than that part of the current which continues to flow through the bend. The change of speed becomes stronger with increasing frequency.

\section{Conclusions}

This paper focused on the question of the physical meaning of the line parameters in the TLST. The meaning of the 


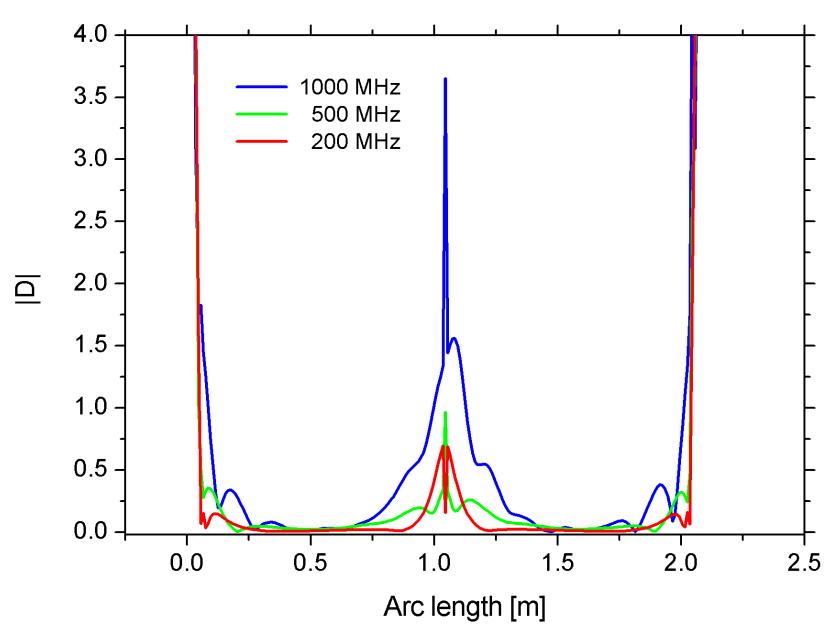

Figure 14. Magnitude of the damping function $|D|$ along the line with central local scatterer for different frequencies $(200,500 \mathrm{MHz}$, $1 \mathrm{GHz})$.

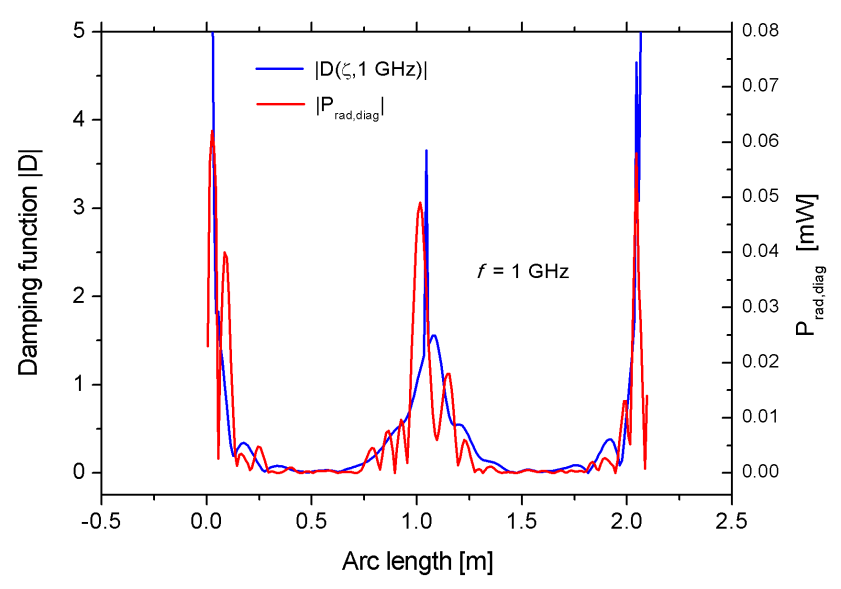

Figure 15. Magnitude of the damping function $|D|$ in comparison with the magnitude of the radiated power from the diagonal elements.

parameters depends on the representation of the TLST equations. In the charge-current representation the two line parameters were directly correlated to measurable quantities: namely, the propagation function $\Gamma$ and the damping function $D$. Therefore, this representation is the most suitable for application. In the case of low frequencies $(k \rightarrow 0)$ there is an algebraic relation between the potential (voltage) and the charge via the capacitive matrix (see Eqs. 9a and 10). Thus, with the aid of Eqs. (6) through (10) the charge-current representation is easily transferred into the potential(voltage)current representation with the parameter matrix $\overline{\mathbf{P}}^{*(0)}(l)$. Here, there are zeros in the diagonal matrix while the offdiagonal matrices represent the (symmetrical) inductance matrix and the (symmetrical) capacitance matrix, respectively. Both matrices have a known physical meaning. The situation changes for high frequencies when radiation occurs.

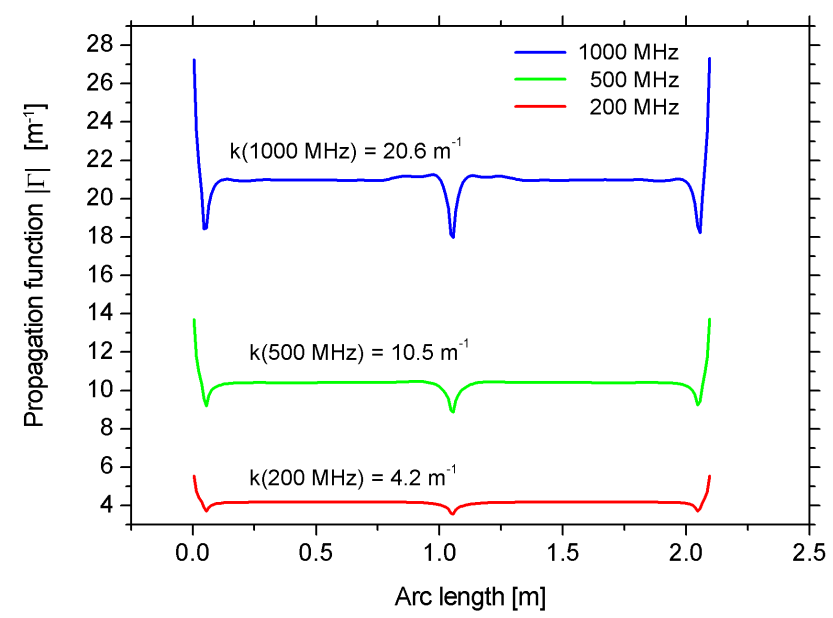

Figure 16. Magnitude of the propagation function $|\Gamma|$ at different frequencies for the parallel line with central local scatterer.

In this case, the former algebraic relation changes to an integral relation (see Eq. 14) and the potential-current equations are given by the more complicated Eqs. (16) and (17a, b). Now the line parameters lose their direct physical meaning and are gauge dependent. Only in combinations they can be related to physical observables, like radiation or propagation function and damping function (Nitsch and Tkachenko, 2005). The more fundamental reason that the parameter elements lose their direct contact to physical observables lies in the fact that forward- and backward-running current waves have (in general) different capacitances and inductances (see Eqs. 26 to 29). This applies both in the Coulomb gauge as well as in the Lorenz gauge. The fact that forward and backward-running current waves have different inductances and capacitances leads to non-symmetrical parameter submatrices $\left(\mathbf{P}_{i j, \text { Lor }}^{*(1)}\right.$ and $\left.\mathbf{P}_{i j, \text { Coul }}^{*(1)}, i, j=1,2\right)$. However, the reference to the quantities inductance and capacitance, known from classical TLT, remains intact. In TLST the parameters are composed of combinations of capacitances and inductances for forward and backward running waves.

Acknowledgements. The authors would like to thank Günter Wollenberg for his permanent interest in our work and for fruitful discussions.

Edited by: F. Gronwald

Reviewed by: three anonymous referees

\section{References}

Gantmacher, F.: The theory of matrices, Chelsea Publishing Company, New York, 1984.

Haase, H. and Nitsch, J.: Full-wave transmission-line theory (FWTLT) for the analysis of three dimensional wire-like struc- 
tures, in: Proc. 14th International Zurich Symposium and Technical Exhibition on Electromagnetic Compatibility, 235-240, Zurich, Switzerland, 2001.

Kazemzadeh, R. and Mathis, W.: Description of Non-Quasistatic Effects Using Partial Elements, in: XV. International Symposium on Theoretical Electrical Engineering (ISTET), 22-24 June 2009, Lübeck, Germany, 2009.

Nitsch, J. and Tkachenko, S.: Complex-Valued Transmission-Line Parameters and Their Relation to the Radiation Resistance, IEEE T. Electromag. C., 46, 477-487, 2004.

Nitsch, J. and Tkachenko, S.: Global and Modal Parameters in the Generalized Transmission-Line Theory and Their Physical Meaning, The Radio Science Bulletin, 312, 21-31, 2005.

Nitsch, J. and Tkachenko, S.: Physical Interpretation of the Parameters in the Full-Wave Transmission-Line Theory, ISTET 2009, 2009.
Nitsch, J. and Tkachenko, S.: High-Frequency Multiconductor Transmission-Line Theory, Found. Phys., 40, 1231-1252, 2010.

Nitsch, J., Gronwald, F., and Wollenberg, G.: Radiating Nonuniform Transmission-Line Systems and the Partial Element Equivalent Circuit Method, Wiley, Chichester, West Sussex, UK, 2009.

Rambousky, R., Nitsch, J., and Tkachenko, S.: Transmission-Line Super Theory as Antenna Theory for Linear Structures, in: UltraWideband, Short-Pulse Electromagnetics 10, edited by: Sabath, F. and Mokole, E., Springer, Berlin, Germany, 13-19, 2014.

Schwark, M., Junge, A., and Kebel, R.: VHF Interference Measurements on Cable Harnesses in Passenger Cabins, IEEE T. Electromag. C., Special Issue on Aerospace EMC, 50, 536-541, 2008.

Shen, W., Xue, C., Mei, K., and Lin, J.: Maxwellian Circuits of Conducting Circular Loops, IEEE Antennas Propag., 59, 38483854, 2011. 